\title{
XIX. On the double achromatic object glass
}

\author{
J.W. Lubbock Esq. V.P. \& Treas. R.S.
}

To cite this article: J.W. Lubbock Esq. V.P. \& Treas. R.S. (1835) XIX. On the double achromatic object glass, Philosophical Magazine Series 3, 7:39, 161-171, DOI: $10.1080 / 14786443508648690$

To link to this article: http://dx.doi.org/10.1080/14786443508648690

$$
\text { 曲 Published online: } 01 \text { Jun } 2009 .
$$

Submit your article to this journal $\widetilde{ }$

\section{Џ Article views: 2}




\title{
LONDON AND EDINBURGH .
}

\section{PHILOSOPHICAL MAGAZINE}

\author{
AN D \\ JOURNAL OF SCIENCE.
}

[THIRD SERIES.]

$S E P T E M B E R \quad 1835$.

XIX. On the Double Achromatic Object Glass. By J. W. Lubвоск, Esq., V.P.\& Treas. R.S.*

IN the Philosophical Transactions for 1821, Sir John F. W. 1 Herschel has entered minutely into the theory of the double achromatic object glass, and has given various useful tables showing the curvatures of the lenses to be employed in order that the spherical and chromatic aberrations may be destroyed + . Sir John Herschel seems to have intended to consider at some future time the modifications which would result in the inquiry when the thicknesses are not neglected, but I am not aware that he ever carried this intention into effect. I have therefore endeavoured to put the expressions which occur in the theory of spherical aberration in the most advantageous form. In this form they admit of extension to the case when the thicknesses of the lenses and the interval between them are considered, without any difficulty.

Whether or not opticians will ever trust in great measure to theory for the curvatures of the lenses, taking care previously to ascertain with precision the optical characters of the glass of which they are composed, may admit of doubt; but it would certainly be useful to know the figures of the lenses and other circumstances connected with any large object glass whose performance is considered perfect, in order

* Communicated by the Author.

+ A notice of Sir John Herschel's paper will be found in Phil. Mag., vol. Ix. p. 147.-Enrt.

Third Series. Vol. 7. No. 39. Sept. 1835. 


\section{Mr. Lubbock on the Double Achromatic Object Glass.}

that these data might be compared with the results given by theory*. Unfortunately no such measures have yet been published, as far as I am aware, except those in Sir David Brewster's Encyclopædia, under the article Achromatic Telescopes; but certain data are there wanting to render them complete. I am informed that the universal practice in this country and in France has been to make the flint lens double concave, and not concavo-convex as theory indicates, which circumstance seems to me very extraordinary. I am told that Fraunhofer employed the latter construction; but it would seem from the representation of an object glass incidentally given in a plate which accompanies his paper on the lines in the spectrum, that he did not do so invariably.

$$
\text { Let } \mathrm{EA} \text { or } \mathrm{ER}=r \quad \mathrm{AQ}=\Delta \quad \mathbf{A} q=\Delta^{\prime} \quad \mathbf{R P}=y
$$

$\mathrm{QR}$ is the incident ray meeting the axis $\mathrm{A} E$ in $\mathrm{Q}, \mathrm{R} q$ the refracted ray, the direction of which cuts the axis in $q$, $\mathbf{E}$ the centre of the surface of which $\mathbf{R A}$ is a section. $\mathbf{R P}$ is drawn perpendicular to $\mathrm{A} \mathrm{E}$. The notation employed is that of Mr. Coddington's Elementary Treatise on Optics, 2nd edit., p. 54.

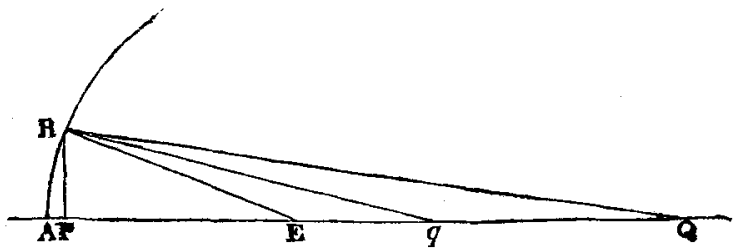

$$
m=\frac{\sin \mathrm{ERQ} \sin \mathrm{RE} q}{\sin \mathrm{REQ} \sin \mathrm{ER} q}=\frac{\mathrm{EQ} \cdot \mathrm{R} q}{\mathrm{RQ} \cdot \mathrm{E} q}=\frac{(\Delta-r) \mathcal{L}\left\{\left(\Delta^{\prime}-\mathrm{AP}\right)^{2}+y^{2}\right\}}{\left(\Delta^{\prime}-r\right) \sqrt{ }\left\{(\Delta-\mathrm{AP})^{2}+y^{2}\right\}}
$$

$m$ being the index of refraction for the substance of which the lens is formed, which is bounded by the sphere of which $\mathbf{E}$ is the centre.

$$
\begin{gathered}
\mathbf{A} \mathbf{P}=\frac{y^{2}}{2 r-\mathbf{A} \mathbf{P}} \quad \mathbf{A} \mathbf{P}=\frac{y^{2}}{2 r}\left(1+\frac{y^{2}}{4 r^{2}}\right) \text { nearly } \\
\left(\Delta^{\prime}-\mathrm{AP}\right)^{2}=\Delta^{\prime 2}-\frac{\Delta^{\prime} y}{r}\left(1+\frac{y^{2}}{4 r^{2}}\right)+\frac{y^{4}}{4 r^{2}}+y^{2}
\end{gathered}
$$

* The curvatures of the surfaces can be ascertained very correctly by the Spherometer, an instrument described by M. Biot. (Traité de Physique, vol. iv. p. 343.) 
Mr. Lubbock on the Double Achromatic Object Glass. 163

$$
\begin{gathered}
=\Delta^{\prime 2}\left\{1-\frac{1}{\Delta^{\prime}}\left(\frac{1}{r}-\frac{1}{\Delta^{\prime}}\right)\left(y^{2}+\frac{y^{4}}{4 r^{2}}\right)\right\} \\
m^{2}\left(\Delta^{\prime}-r\right)^{2} \Delta^{2}\left\{1-\frac{1}{\Delta}\left(\frac{1}{r}-\frac{1}{\Delta}\right)\left(y^{2}+\frac{y^{4}}{4 r^{2}}\right)\right\} \\
=(\Delta-r)^{2} \Delta^{\prime 2}\left\{1-\frac{1}{\Delta^{\prime}}\left(\frac{1}{r}-\frac{1}{\Delta^{\prime}}\right)\left(y^{2}+\frac{y^{4}}{4 r^{2}}\right\}\right) \\
\frac{1}{r}-\frac{1}{\Delta^{\prime}}=\frac{1}{m}\left\{\frac{1}{r}-\frac{1}{\Delta}\right\}+\left(\frac{1}{r}-\frac{1}{\Delta}\right)\left(\frac{1}{r}-\frac{1}{\Delta}\right)\left(\frac{1}{\Delta}-\frac{1}{m \Delta^{\prime}}\right) \frac{y^{3}}{2} \\
+\left(\frac{1}{r}-\frac{1}{\Delta^{\prime}}\right)\left(\frac{1}{r}-\frac{1}{\Delta}\right)\left\{\frac { 1 } { \Delta } \left(\frac{1}{r^{2}}+\frac{1}{\Delta}\left(\frac{1}{r}-\frac{1}{\Delta}\right)\right.\right. \\
-\frac{1}{m^{\prime} \Delta}\left(\frac{1}{r^{2}}+\frac{1}{\Delta}\left(\frac{1}{r}-\frac{1}{\Delta^{\prime}}\right)\right\} \frac{y^{4}}{8}+, \& \mathrm{c} .
\end{gathered}
$$

Neglecting $y^{q}$,

$\frac{1}{r}-\frac{1}{\Delta}=\frac{m}{m-1}\left(\frac{1}{\Delta^{\prime}}-\frac{1}{\Delta}\right) \quad \frac{1}{r}-\frac{1}{\Delta^{\prime}}=\frac{1}{m-1}\left(\frac{1}{\Delta^{\prime}}-\frac{1}{\Delta}\right)$.

Hence, neglecting $y^{4}$,

$$
\frac{1}{\Delta^{\prime}}=\frac{m-1}{m r}+\frac{1}{m \Delta}+\left\{\frac{1}{m \Delta^{\prime}}-\frac{1}{\Delta}\right\}\left\{\frac{1}{\Delta^{\prime}}-\frac{1}{\Delta}\right\}^{2} \frac{m y^{2}}{2(m-1)^{2}} .
$$

Hence, if $r_{1}, r_{2}, r_{3}, r_{4}$ are the radii of four spherical surfaces; $\Delta_{1}, \Delta_{2}, \Delta_{3}, \Delta_{4}$, the distances from the vertex at which the ray intersects their common axis after refraction at the first, second, third, and fourth surfaces respectively, neglecting the thicknesses; $m$, the index of refraction for the substance of which the lens is formed which is bounded by the first and second surfaces; $m^{\prime}$, the index of refraction for the substance of which the lens is formed which is bounded by the third and fourth surfaces; then, neglecting $y^{4}$,

$$
\begin{aligned}
& \frac{1}{\Delta}=\frac{m-1}{m r}+\frac{1}{m \Delta}+\left\{\frac{1}{m \Delta_{1}}-\frac{1}{\Delta}\right\}\left\{\frac{1}{\Delta_{1}}-\frac{1}{\Delta}\right\}^{2} \frac{m y^{2}}{2(m-1)^{2}} \\
& \frac{1}{\Delta_{2}}=-\frac{m-1}{r_{2}}+\frac{m}{\Delta_{1}}+\left\{\frac{1}{\Delta_{2}}-\frac{1}{m \Delta_{1}}\right\}\left\{\frac{1}{\Delta_{2}}-\frac{1}{\Delta_{1}}\right\}^{2} \frac{m^{2} y^{2}}{2(m-1)^{2}} \\
& \frac{1}{\Delta_{9}}=\frac{m^{\prime}-1}{r_{3}}+\frac{1}{m^{\prime} \Delta_{2}}+\left\{\frac{1}{m^{\prime} \Delta_{3}}-\frac{1}{\Delta_{2}}\right\}\left\{\frac{1}{\Delta_{9}}-\frac{1}{\Delta_{2}}\right\}^{2} \frac{m^{\prime} y^{2}}{2\left(m^{\prime}-1\right)^{2}} \\
& \frac{1}{\Delta_{4}}=-\frac{m^{\prime}-1}{r_{4}}+\frac{m^{\prime}}{\Delta_{3}}+\left\{\frac{1}{\Delta_{4}}-\frac{1}{m^{\prime} \Delta_{3}}\right\}\left\{\frac{1}{\Delta_{4}}-\frac{1}{\Delta_{3}}\right\}^{2} \frac{m^{2} y^{2}}{2\left(m^{\prime}-1\right)^{2}} \\
& \frac{1}{\Delta_{4}}=-\left(m^{\prime}-1\right)\left\{\frac{1}{r_{4}}-\frac{1}{r_{3}}\right\}-(m-1)\left\{\frac{1}{r_{2}}-\frac{1}{r_{1}}\right\}+\frac{1}{\Delta} \\
& Y 2
\end{aligned}
$$


164 Mr. Lubbock on the Double Achromatic Object Glass.

$+\left\{\left\{\frac{1}{m \Delta_{1}}-\frac{1}{\Delta}\right\}\left\{\frac{1}{\Delta_{l}}-\frac{1}{\Delta}\right\}^{2}+\left\{\frac{1}{\Delta_{2}}-\frac{1}{m \Delta_{1}}\right\}\left\{\frac{1}{\Delta^{2}}-\frac{1}{\Delta_{1}}\right\}^{2}\right\} \frac{m^{2} y^{2}}{2(m-1)}$ $+\left\{\left\{\frac{1}{m^{\prime} \Delta_{9}}-\frac{1}{\Delta_{q}}\right\}\left\{\frac{1}{\Delta_{3}}-\frac{1}{\Delta_{q}}\right\}^{2}+\left\{\frac{1}{\Delta_{4}}-\frac{1}{m^{\prime} \Delta_{9}}\right\}\left\{\frac{1}{\Delta_{4}}-\frac{1}{\Delta_{3}}\right\}^{2}\right\} \frac{m^{\prime 2} y^{2}}{2\left(m^{\prime}-1\right)^{2}}$

This expression for $\frac{1}{\Delta_{4}}$ may be put into the following more convenient form :

$$
\begin{aligned}
& \frac{1}{\Delta_{4}}=-\left(m^{\prime}-1\right)\left\{\frac{1}{r_{4}}-\frac{1}{r_{9}}\right\}-(m-1)\left\{\frac{1}{r_{2}}-\frac{1}{r_{1}}\right\}+\frac{1}{\Delta} \\
& +\left(\frac{1}{\Delta_{2}}-\frac{1}{\Delta}\right)\left\{\left(\frac{1}{\Delta_{2}}-\frac{1}{\Delta_{1}}\right)^{2}+\left(\frac{1}{\Delta}-\frac{1}{m \Delta_{1}}\right)\left(\frac{1}{\Delta}-\frac{2}{\Delta_{1}}+\frac{1}{\Delta}\right)\right\} \frac{m^{3} y^{2}}{2(m-1)^{2}} \\
& +\left(\frac{1}{\Delta_{4}}-\frac{1}{\Delta_{2}}\right)\left\{\left(\frac{1}{\Delta_{4}}-\frac{1}{\Delta_{9}}\right)^{2}+\left(\frac{1}{\Delta_{2}}-\frac{1}{m^{\prime} \Delta_{9}}\right)\left(\frac{1}{\Delta_{4}}-\frac{2}{\Delta_{9}}+\frac{1}{\Delta_{2}}\right)\right\} \frac{m^{13} y^{2}}{2\left(m^{\prime}-1\right)}
\end{aligned}
$$

In order to get rid of spherical aberration, the quantities $\Delta_{1}, \Delta_{2}, \Delta_{3}, \Delta_{4}$ must be determined by the condition that the coefficient of $y^{2}$ in the last equation $=$ zero. In the construction recommended by Sir John Herschel, the object glass consists of a double-convex lens of crown glass and a concavoconvex lens of flint glass. This combination is represented in the figure underneath, where the four surfaces are numbered

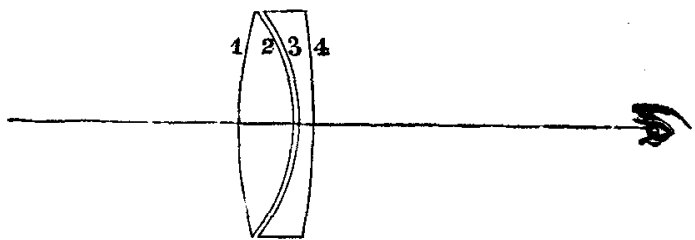

in the order in which the light traverses them in its passage from the object to the eye.

Neglecting $y^{z}$, if

$$
\begin{gathered}
\frac{1}{\Delta_{1}}=f_{1}+\frac{1}{m \Delta} \quad \frac{1}{\Delta_{2}}=f_{2}+\frac{1}{\Delta} \\
\frac{1}{\Delta_{3}}=f_{3}+\frac{1}{m^{\prime} \Delta} \quad \frac{1}{\Delta_{4}}=f_{4}+\frac{1}{\Delta},
\end{gathered}
$$

and if $\varpi$ be the ratio of the dispersive power of the crown glass to the dispersive power of the flint, it is easy to show that the condition of achromaticity requires

$$
\frac{1}{\Delta_{4}}-\frac{1}{\Delta_{2}}=-\approx\left\{\frac{1}{\Delta_{2}}-\frac{1}{\Delta}\right\}
$$



or, for parallel rays, that is, when $\Delta$ is infinite and $\frac{1}{\Delta}=0$,

$$
\frac{1}{\Delta_{4}}-\frac{1}{\Delta}=-\frac{\varpi}{\Delta_{2}} \quad f_{2}=\frac{f_{4}}{1-\sigma}
$$

$f_{4}$ may be assumed at pleasure, and $f_{2}$ being obtained from the last equation, $f_{2}$ and $f_{4}$ may be considered as given. It remains to determine $f_{1}, f_{3}$, in doing which another arbitrary condition is admissible.

$$
\text { If } \begin{aligned}
& \approx \frac{m^{18}(m-1)^{8}}{\left(m^{\prime 2}-1\right)^{2} m^{2}}=\lambda, \text { for parallel rays, } \\
& \left(f_{2}-f_{1}\right)^{8}-\frac{f_{1}}{m}\left(f_{2}-2 f_{1}\right) \\
= & \lambda\left\{\left\{f_{4}-f_{3}\right\}^{8}+\left\{f_{2}-\frac{f_{3}}{m}\right\} \cdot\left\{f_{4}-2 f_{3}+f_{2}\right\}\right\} .
\end{aligned}
$$

The last equation coincides with the equation $(z)$ of Sir John Herschel in the Phil. Trans. for 1821, p. 258.

Since

$$
\begin{array}{ll}
\frac{1}{\Delta_{2}}=-\frac{m-1}{r_{2}}+\frac{m}{\Delta_{1}} & \frac{1}{r_{2}}=\frac{m}{m-1}\left\{\frac{1}{\Delta_{1}}-\frac{1}{m \Delta_{2}}\right\} \\
\frac{1}{\Delta_{3}}=\frac{m^{\prime}-1}{m^{\prime} r_{3}}+\frac{1}{m^{\prime} \Delta_{2}} & \frac{1}{r_{3}}=\frac{m^{\prime}}{m^{\prime}-1}\left\{\frac{1}{\Delta_{3}}-\frac{1}{m^{\prime} \Delta_{2}}\right\}
\end{array}
$$

if the radii of the second and third surfaces are equal

$$
\begin{aligned}
& \frac{m}{m-1}\left\{\frac{1}{\Delta_{1}}-\frac{1}{m \Delta_{2}}\right\}=\frac{m^{\prime}}{m^{\prime}-1}\left\{\frac{1}{\Delta_{3}}-\frac{1}{m^{\prime} \Delta_{2}}\right\} \\
& \frac{m}{m-1}\left\{f_{1}-\frac{f_{2}}{m}\right\}=\frac{m^{\prime}}{m^{\prime}-1}\left\{f_{3}-\frac{f_{2}}{m^{\prime}}\right\} .
\end{aligned}
$$

This condition was originally suggested by Clairaut; but according to Sir John Herschel, "when the average values of the indices of refraction, such as are likely to occur most frequently, are employed, the construction becomes imaginary for the more dispersive kinds of glass; and within the limits for which it is real, the radii change so rapidly as to render it difficult to interpolate between their calculated values."

Hence, instead of this condition, Sir John Herschel recommends another, which results from making equal to zero the coefficient of $\frac{1}{\Delta}$ in the quantity 
166 Mr. Lubbock on the Double Achromatic Object Glass.

$$
\begin{aligned}
& \left\{\frac{1}{\Delta_{2}}-\frac{1}{\Delta_{1}}\right\}^{2}+\left\{\frac{1}{\Delta_{2}}-\frac{1}{m \Delta_{1}}\right\}\left\{\frac{1}{\Delta_{2}}-\frac{2}{\Delta_{1}}+\frac{1}{\Delta}\right\}^{2} \\
= & \lambda\left\{\frac{1}{\Delta_{4}}-\frac{1}{\Delta_{3}}\right\}^{2}+\left\{\frac{1}{\Delta_{2}}-\frac{1}{m^{\prime} \Delta_{3}}\right\}\left\{\frac{1}{\Delta_{4}}-\frac{2}{\Delta_{3}}+\frac{1}{\Delta_{2}}\right\}^{2},
\end{aligned}
$$

in order that the telescope may be adapted for seeing nearer as well as more remote objects. This condition gives

$$
\begin{gathered}
\frac{2(m-1)}{m}\left\{f_{2}-f_{1}\right\}+\frac{(m-1)(m+1)}{m^{2}}\left\{f_{2}-2 f_{1}\right\}-\frac{2(m-1)}{m} f_{1} \\
=\lambda\left\{\frac{2\left(m^{\prime}-1\right)}{m^{\prime}}\left\{f_{4}-f_{3}\right\}+\frac{\left(m^{\prime}-1\right)\left(m^{\prime}+1\right)}{m^{\prime 2}}\left\{f_{4}-2 f_{3}+f_{2}\right\}\right. \\
\left.+\frac{2\left(m^{\prime}-1\right)}{m^{\prime}}\left\{f_{2}-\frac{1}{m^{\prime}} f_{3}\right\}\right\} .
\end{gathered}
$$

The last equation coincides with the equation (A) of Sir John Herschel, p. 258. This equation is to be combined with the equation, p. 165 , line 8 , which may be put into the form

$$
\begin{gathered}
f_{1}^{2}-f_{2}\left(\frac{2 m+1}{m+2}\right) f_{1}+\frac{m}{m+2} f_{2}^{2} \\
=\lambda \frac{m\left(m^{\prime}+2\right)}{(m+2) m^{\prime}}\left\{f_{3}^{2}-\left(f_{4}+f_{2}\right)\left(\frac{2 m^{\prime}+1}{m^{\prime}+2}\right) f_{3}\right. \\
+\frac{m^{\prime}}{m^{\prime}+2}\left\{f_{4}^{2}+f_{2}\left(f_{4}+f_{2}\right)\right\} .
\end{gathered}
$$

Having found $f_{1}, f_{2}, f_{3}, f_{4}$, the radii of curvature may be obtained by means of the equations

$$
\begin{aligned}
& f_{1}=\frac{m-1}{m r_{1}} \quad f_{2}=-\frac{m-1}{r_{2}}+m f_{1} \\
& f_{3}=\frac{m^{\prime}-1}{m^{\prime} r_{3}}+\frac{f_{2}}{m^{\prime}} \quad f_{4}=-\frac{\left(m^{\prime}-1\right)}{r_{4}}+m^{\prime} f_{3} .
\end{aligned}
$$

If $m=1.524, \quad m^{\prime}=1.585, \quad \varpi=\cdot 60$, (which are the average values, Sir John Herschel finds, assuming $f_{4}=-\cdot 1$, and with the conditions which he recommends,

$$
\begin{array}{cccc}
r_{1}=-6.7069 & r_{2}=3.0488 & r_{3}=3.0640 & r_{4}=14.2937 . \\
\multicolumn{4}{c}{\text { With these curvatures }} \\
f_{1}=-.051265 & f_{2}=-.25 & f_{3}=-.03727 & f_{4}=-\cdot 1 \\
f_{2}-f_{1}=-.19873 & -\frac{f_{1}}{m}=.03364 & f_{2}-2 f_{1}=-.14746
\end{array}
$$


Mr. Lubbock on the Double Achromatic Object Glass. 167

$f_{4}-f_{3}=-06272 \quad f_{2}-\frac{f_{3}}{m^{\prime}}=-22648 \quad f_{4}-2 f_{3}+f_{2}=-\cdot 27544$

$-\frac{f_{1}{ }^{3}}{m}=-.0000884 \quad\left(f_{2}-\frac{f_{1}}{m}\right)\left(f_{2}-f_{1}\right)^{2}=-\cdot 0085448$

$\left(\frac{f_{3}}{m}-f_{2}\right)\left(f_{3}-f_{2}\right)^{2}=\cdot 010249 \quad\left(f_{4}-\frac{f_{3}}{m^{\prime}}\right)\left(f_{4}-f_{3}\right)^{2}=-\cdot 000301$.

The preceding values will be found to destroy the coeffcient of $y^{2}$ in the expression for $f_{4}$. The values of the radii are taken from a very useful table given by Sir John Herschel in the paper before alluded to.

One advantage which seems to me to result from the form into which the equations have been put in this short view of the theory of aberration is the facility with which the thicknesses of the lenses and the interval between them can be taken into account. For if $\Delta_{1}, \Delta_{2}, \Delta_{3}, \Delta_{4}$, are measured, as before, from the points at which the surfaces cut the axis respectively, we have obviously

$\frac{1}{\Delta_{1}}=\frac{m-1}{m r_{1}}+\frac{1}{m \Delta}+\left\{\frac{1}{m \Delta_{1}}-\frac{1}{\Delta}\right\}\left\{\frac{1}{\Delta_{1}}-\frac{1}{\Delta}\right\}^{2} \frac{m y^{8}}{2(m-1)^{2}}$, or, for parallel rays,

$$
\frac{1}{\Delta_{1}}=\frac{m-1}{m r_{1}}+\frac{y^{2}}{2 \Delta_{1}^{3}(m-1)^{2}}
$$

and if $t_{1}$ be the thickness of the anterior lens, or the interval between the intersections of the axis by the first and second surfaces,

$$
\begin{aligned}
& \frac{1}{\Delta_{2}}=-\frac{m-1}{r_{2}}+\frac{m}{\Delta_{1}+t_{1}} \\
& +\left\{\frac{1}{\Delta_{2}}-\frac{1}{m\left(\Delta_{1}+t_{1}\right)}\right\}\left\{\frac{1}{\Delta_{2}}-\frac{1}{\Delta_{1}+t_{1}}\right\} \frac{\left(\Delta_{1}+t_{1}\right)^{2} m^{2} y^{2}}{2 \Delta_{1}^{2}(m-1)^{2}} .
\end{aligned}
$$

In Sir John Herschel's construction the interval between the lenses may be made inconsiderable. In this case

$$
\begin{aligned}
& \frac{1}{\Delta_{3}}=\frac{m^{\prime}-1}{m^{\prime} r_{3}}+\frac{1}{m^{\prime} \Delta_{3}} \\
& +\left\{\frac{1}{m^{\prime} \Delta_{2}}-\frac{1}{\Delta_{2}}\right\}\left\{\frac{1}{\Delta_{3}}-\frac{1}{\Delta_{2}}\right\}^{2} \frac{m^{\prime}\left(\Delta_{1}+t_{1}\right)^{2} y^{2}}{2 \Delta_{1}^{2}\left(m^{\prime}-1^{2}\right.}
\end{aligned}
$$

and if $t_{2}$ be the thickness of the second lens,

$$
\begin{aligned}
& \frac{1}{\Delta_{4}}=-\frac{m^{\prime}-1}{r_{4}}+\frac{m}{\Delta_{3}+t_{2}} \\
& +\left\{\frac{1}{\Delta_{4}}-\frac{1}{m^{\prime}\left(\Delta_{3}+t_{2}\right)}\right\}\left\{\frac{1}{\Delta_{4}}-\frac{1}{\Delta_{3}+t_{2}}\right\}^{2} \frac{m^{\prime 2}\left(\Delta_{1}+t_{1}\right)^{2}\left(\Delta_{3}+t_{2}\right)^{2} y^{8}}{2 \Delta_{1}^{2} \Delta_{3}^{8}\left(m^{\prime}-1\right)^{z}} .
\end{aligned}
$$


168 Mr. Lubbock on the Double Achromatic Object Glass.

In this construction the thickness of the second lens may also be made inconsiderable, and as $\frac{1}{\Delta_{4}}-\frac{1}{m^{\prime} \Delta_{3}}$ and $\frac{1}{\Delta_{4}}-\frac{1}{\Delta_{3}}$ are small quantities, (see the numerical values, p. 167,) $t_{2}$ may certainly be disregarded in the cuefficient of $y^{2}$ in the expression for $\frac{1}{\Delta_{4}}$.

Neglecting $y^{2}$ and $\frac{1}{\Delta}$,

$$
\begin{gathered}
f_{2}=\frac{1}{\Delta_{2}}=-(m-1)\left\{\frac{1}{r_{2}}-\frac{1}{r_{1}}\right\}-\frac{m t_{1}}{\Delta_{1}^{8}} \\
f_{4}=\frac{1}{\Delta_{4}}=-\left(m^{\prime}-1\right)\left\{\frac{1}{r_{4}}-\frac{1}{r_{3}}\right\}-(m-1)\left\{\frac{1}{r_{2}}-\frac{1}{r_{1}}\right\} \\
-\frac{m^{\prime} t_{2}}{\Delta_{3}^{2}}-\frac{m t_{1}}{\Delta_{1}^{2}} \delta_{m^{\prime}}\left\{\frac{1}{r_{4}}-\frac{1}{r_{3}}+\frac{t_{2}}{\Delta_{3}^{8}}\right\}+\delta m\left\{\frac{1}{r_{2}}-\frac{1}{r_{1}}+\frac{t_{1}}{\Delta_{1}^{2}}\right\}=0 \\
f_{4}-f_{2}+\frac{t_{2}}{\Delta_{3}^{8}}=-\frac{\frac{\partial m}{m-1}}{\delta m^{\prime}}\left\{f_{2}+\frac{t_{1}}{\Delta_{1}^{2}}\right\} \\
\left.=-\varpi f_{2}+\frac{t_{1}}{\Delta_{1}^{8}}\right\} \\
f_{4}=(1-\varpi) f_{2}-\frac{\varpi t_{1}}{\Delta_{1}^{2}} .
\end{gathered}
$$

In the numerical example given, p. 166, supposing the aperture $=1$, and the thickness of the double-convex lens to vanish at the circumference of the field, $t_{1}=006$ nearly.

$$
\begin{gathered}
f_{3}^{2}-\frac{\left(2 m^{\prime}+1\right)}{\left(m^{\prime}+2\right)}\left(f_{4}+f_{2}\right) f_{3}+\frac{m^{\prime}}{m^{\prime}+2}\left\{f_{4}^{2}+f_{2}\left(f_{4}+f_{2}\right)\right\} \\
+\frac{m^{2}\left(m^{\prime}-1\right)^{2}}{\left(f_{4}-f_{2}\right)\left(m^{\prime}+2\right) m^{\prime}(m-1)^{2}}\left\{\left(\frac{1}{\Delta_{2}}-\frac{1}{m\left(\Delta_{1}+t_{1}\right)}\right)\right. \\
\left.\quad\left(\frac{1}{\Delta_{2}}-\frac{1}{\Delta_{1}+t_{1}}\right)^{2}+\frac{1}{m \Delta_{1}\left(\Delta_{1}+t_{1}\right)^{2}}\right\}=0 .
\end{gathered}
$$

I find, (retaining the previous values of $r_{1}, r_{2}$ ) see p. 166, that is, making $r_{1}=-6.7069 \quad r_{2}=3.0488$

$$
\begin{aligned}
& \left.\frac{1}{\Delta_{1}}=-.051265\right) \\
& f_{2}=\frac{1}{\Delta_{2}}=-.25024 \quad f_{4}=\frac{1}{\Delta_{4}}=-\cdot 10023
\end{aligned}
$$


Mr. Lubbock on the Double Achromatic Object Glass. 169 from the conditions of achromaticity and the equation to determine $f_{3}$, after the numerical substitutions, is

$$
\begin{aligned}
& f_{3}^{8}+\cdot 40766 f_{3}=-.01377 \text {, which equation gives } \\
& f_{3}=-.03716 \text { or }-.37050 \text {. } \\
& \text { From } f_{3}=-.03716 \text {, I find } \\
& \quad r_{3}=3.0573 \text { and } r_{4}=14.151,
\end{aligned}
$$

instead of $r_{3}=3.0640$ and $r_{4}=14.297$, which were the values of those quantities when the thickness of the donbleconvex lens was neglected. The difference in the values of the radii corresponds in each case to the indication 0007 inch by a spherometer of 5 -inch radius, which might be appreciable in large telescopes, but not, 1 apprehend, in common in. struments.

In the last example I have neglected the interval between the lenses : if this be taken into account and the thicknesses of the lenses be neglected, it is evident that for parallel rays

$$
\begin{aligned}
& \frac{1}{\Delta_{1}}=\frac{m-1}{m r_{1}}+\frac{y^{2}}{2 \Delta_{1}^{3}(m-1)^{2}} \\
& \frac{1}{\Delta_{2}}=-\frac{m-1}{r_{2}}+\frac{m}{\Delta_{1}}+\left\{\frac{1}{\Delta^{2}}-\frac{1}{m \Delta_{1}}\right\}\left\{\frac{1}{\Delta_{2}}-\frac{1}{\Delta_{1}}\right\}^{2} \frac{m^{2} y^{3}}{2(m-1)_{6}} .
\end{aligned}
$$

If the interval between the lenses be called $t_{2}$,

$$
\begin{aligned}
& \frac{1}{\Delta_{3}}=\frac{m^{\prime}-1}{m^{\prime} r_{3}}+\frac{1}{m^{\prime}\left(\Delta_{2}+t_{2}\right)} \\
& +\left\{\frac{1}{m^{\prime} \Delta_{3}}-\frac{1}{\Delta_{2}+t_{2}}\right\}\left\{\frac{1}{\Delta_{3}}+\frac{1}{\Delta_{2}+t_{2}}\right\}^{8} \frac{m^{\prime}\left(\Delta_{2}+t_{2}\right)^{2}}{2 \Delta_{2}^{2}\left(m^{\prime}-1\right)^{2}} y^{2} \\
& \frac{1}{\Delta_{4}}=-\frac{m^{\prime}-1}{r_{4}}+\frac{m^{\prime}}{\Delta_{3}} \\
& +\left\{\begin{array}{l}
1 \\
\Delta_{4}
\end{array}-\frac{1}{m^{\prime} \Delta_{9}}\right\}\left\{\frac{1}{\Delta_{4}}-\frac{1}{\Delta_{3}}\right\}^{2} \frac{m^{\prime 2}\left(\Delta_{2}+t_{2}\right)^{2}}{2 \Delta_{2}^{2}\left(m^{\prime}-1\right)^{2}} y^{2} .
\end{aligned}
$$

The condition of achromaticity gives

$$
\frac{1}{\Delta_{4}}-\frac{1}{\Delta_{2}}=-\frac{\sigma}{\Delta_{2}}-t_{2}\left\{\frac{1}{\Delta_{2}^{2}}-\frac{1}{\Delta_{3}^{2}}\right\} \text {. }
$$

Retaining the previous curvatures of the flint lens, and the values of $\frac{1}{\Delta_{2}}$ and $\frac{1}{\Delta_{3}}$ in the term multiplied by $t_{2}$, it is easy to calculate the influence which the consideration of the interval between the lenses has upon the curvatures which should be given to the double-convex lens.

Third Series. Vol. 7. No. 39. Sept. 1835. 
Mr. Lubbock on the Double Achromatic Object Glass.

$$
\begin{gathered}
\frac{1}{\Delta_{2}}=\frac{1}{1-\varpi}\left\{\frac{1}{\Delta_{4}}+t_{2}\left(\frac{1}{\Delta_{2}^{2}}-\frac{1}{\Delta_{3}^{2}}\right)\right\} \\
f_{1}^{2}-\frac{(2 m+1)}{m+2} f_{2} f_{1}+\frac{m}{m+2} f_{2}^{z} \\
+\frac{m^{\prime 2}(m-1)^{2}\left(\Delta_{2}+t_{2}\right)^{2}}{f_{2}(m+2) m\left(m^{\prime}-1\right)^{8} \Delta_{2}^{2}}\left\{\left(\frac{1}{m^{\prime} \Delta_{3}}-\frac{1}{\Delta_{2}+t_{2}}\right)\left(\frac{1}{\Delta_{3}}-\frac{1}{\Delta_{2}+t_{2}}\right)^{2}\right. \\
\left.+\left(\frac{1}{\Delta_{4}}-\frac{1}{m^{\prime} \Delta_{3}}\right)\left(\frac{1}{\Delta_{4}}-\frac{1}{\Delta_{3}}\right)^{8}\right\}=0 .
\end{gathered}
$$

Supposing $t_{2}=\cdot 04$, or about half an inch, $\frac{1}{\Delta_{4}}=-\cdot 1$.

I find $\frac{1}{\Delta_{2}}=f_{2}=-.24 .389 \quad \frac{1}{\Delta_{3}}=f_{3}=-.03494$;

and after the numerical substitutions the equation for determining $f_{1}$ becomes

$$
f_{1}^{2}-\cdot 28015 f_{1}=-\cdot 012113,
$$

which equation gives

$f_{1}=-\cdot 05348$, instead of $-\cdot 051265$, which was the value when the interval between the lenses was neglected.

With this value of $f_{1}$, I find

$$
\text { instead of } \quad \begin{aligned}
& r_{1}=6.429 \text { and } r_{2}=3.2868, \\
& r_{1}=6.7069 \text { and } r_{2}=3.0488 . \quad \text { See p. } 168 \text {. }
\end{aligned}
$$

It appears to result, therefore, from calculation, that the interval between the lenses influences materially the performance of the telescope, which indeed is well known to be the case practically. In consequence it would, I think, be desirable to possess a table similar to Sir John Herschel's Table 4. Phil. Trans. 1821, p. 261, calculated for some given interval between the lenses, as half an inch, for instance; such a table, combined with that which Sir John Herschel has given, would afford the means of interpolating for any interval likely to occur in practice, and hence of obtaining in any case accurate values of the curvatures which ought to be given to the four surfaces of the lenses which compose the object glass.

In $\mathrm{p}: 163$, I have left the term multiplied by $y^{4}$, with a view to facilitate the appreciation of the magnitude of this quantity. in the amount of aberration. In order to ascertain what aperture a telescope will bear before this term introduces confusion into the image, it would be necessary previously to know what variation in the focal length (or aberration) the eye 
takes cognisance of. In a telescope of ten feet focal length and an aperture of one foot, a variation of a hundredth of an inch in $\Delta_{4}$ corresponds to 0011 in the coefficient of $\frac{y^{4}}{8}$ in the expression for $\frac{1}{\Delta_{4}}$, and to 00006 in the coefficients of $\frac{y^{8}}{2}$.

XX. A Sketch of the Geology of West Norfolk. By C. B. Rose, Fellow of the Royal Medical and Chirurgical Society of London,"*

THE geological structure of West Norfolk has received but little attention: it was first examined by the celebrated Mr. William Smith, many years since, during his professional engagements in the county. The result of his researches was made public by the appearance, in 1819, of his geological map and section of the county; and were I not in this place to bear willing testimony to the accuracy with which he has laid down the course of the chalk range, and the outcrop of the subjacent strata, I should be committing an act of injustice to one who, from the originality of his views and the great talents for observation he has displayed, is by universal consent acknowledged to be the father of modern geology.

Some distinguished members of the Geological Society have at different times visited this portion of the county, the chief object of attraction having been the cliff at Hunstanton, the only natural section of the regular strata which we possess. The information resulting from these visitations has been, I believe, (not having been able to find anything further, confined within the limits of a note by the Rev. W. D. Conybeare, describing Hunstanton Cliff, in the "Outlines of the Geology of England and Wales'; a description and section of that cliff by Mr. R. C. Taylor in the Philosophical Magazine for February 1823 (vol. lxi. p. 81); and a notice of the chalk of West Norfolk, in a paper read before the Geological Society, May 2nd, 1823 , by the same gentleman, and published in its Transactions. There is also a brief notice of the strata, with a list of the organic remains, in the 'Outlines of the Geology of Norfolk', published in 1833, by Mr. Samuel Woodward of Norwich.

The observations which follow are offered as contribution towards the completion of a task, which, I trust, at some future time will be undertaken by those who are more competent, and have more leisure than myself. 